\title{
Synthesis and modification of a mesoporous material type MCM-41 by an amine for the adsorption of organic pollutants: anionic and cationic dyes.
}

\author{
BENYOUB Nassima ${ }^{1}$, BENHAMOU Abdallah ${ }^{1}$, DEBAB Abdelkader ${ }^{1}$ \\ 1 Departement of Chemical Engineering, Faculty of Chemistry, University of Scinece and Technology \\ MOHAMED BOUDIAF -ORAN, El Mnaouar, BP 1505, Bir El Djir 31000
}

\section{A B $\mathbf{S}$ T $\mathbf{R}$ A $\mathbf{C}$ T}

The main objective of this work was to synthesize the MCM-41 material from optimized protocols. In the second step, the pore size and the specific surface area of the parent material was increased by the incorporation of swelling agents (long chain carbon amines N-N, Dimethyl-dodecylamine «DMDDA ») in post-synthesis. Then the selective extraction of the amine and the calcination allowed us to obtain materials with pore sizes and even larger surfaces than those of the starting materials (parent materials). The adsorbents, identified as MCM-41 (P), DMDDA-41 (A), DMDDA-41 (B), DMDDA-41 (C) and MCM-41 (P/C), were characterized regarding their texture, mesoscopic ordering and chemical surface and finally their adsorption capacity was evaluated using two diffrent dyes (anionic: Orange II sodium and cationic: Janus Green B). The results obtained during the adsorption study show the efficiency of these materials, in particular the "amine and calcined" materials for decolorizing aqueous media contaminated with organic dyes. The kinetic studies and the adsorption isotherms were carried out to clarify the method of fixing each of the two dyes on the two materials tested. The experiments showed that the amine material had a maximum capacity for fixing Orange II (221.06 mg / g) (anionic dye), whereas for a maximum adsorption capacity (455.23 mg / g ) Of the Janus GB (cationic dye), the calcined material is more efficient.

Keywords: Mesoporous materials, MCM-41, adsorption, Orange II Sodium, Janus Green B, adsorption of dyes 


\section{1- Introduction :}

Since the 1990s, the chemistry of inorganic nanostructured materials has developed considerably with the introduction of soft sol-gel chemistry. Thus, the scientists of the Mobil company proposed the first syntheses of mesostructured silicates, ie materials with an organized porous system made up of mesopores [1, 2]. Since then, many research groups have patented new families of materials with different pore structures, pore sizes and synthesis methods: several methods are needed in the manufacturing process of new porous materials organized "MPO".

At present, a new family of ordered mesoporous solids is very widely studied by many researchers from different horizons for various applications including adsorption [3, 4] and catalysis. In the field of adsorption, mesoporous materials such as MCM-41, HMS, SBA-15, SBA-1 have been functionalized by various groups for the adsorption of metal ions and various organic pollutants (synthetic dyes) [5]. The main objective of this work is to synthesize a mesoporous material of type MCM-41 (hexagonal), to modify it by: addition of amine, selective extraction with ethanol and calcination and then $\mathrm{d}$ To evaluate the adsorption capacity of these mesoporous materials with respect to two dyes, anionic sodium orange II and Janus Green B with a cationic character. A detailed study is presented defining the different methods of characterization, as well as a kinetic study and that of adsorption isotherms will also be discussed.L'objectif principal de ce

\section{2- Synthesis and Characterization}

\subsection{Materials :}

The principal reagents used are summarized in a table which follows, where their origin, formula and possibly their purity are specified (Table 1).

In all cases, the solvent employed is distilled water and, the syntheses are carried out at ambient temperature, in an open container. All are made with stirring.

\subsection{S ynthesis:}

In a beaker, the water / TMAOH mixture is stirred vigorously for 5 minutes, then the CTAB is added in small quantities without stopping the stirring which will continue for 30 minutes, and finally the SiO.sub.2 is added in order to have A gel after about 2 hours (the speed of agitation is less strong).

Table 1: Main reagents used

\begin{tabular}{|l|l|l|}
\hline \multicolumn{1}{|c|}{ Reagents } & \multicolumn{1}{c|}{ Formula } & \multicolumn{1}{c|}{ Origin } \\
\hline Cetyltrimethylammonium bromide 99\% $(\mathrm{CTAB})$ & $\mathrm{C}_{19} \mathrm{H}_{42} \mathrm{Br}$ & Biochem \\
\hline Smoked silica 98\% & $\mathrm{SiO}_{2}$ & Alfa Aesar \\
\hline Tetramethylammonium hydroxide (TMAOH) & $\left(\mathrm{CH}_{3}\right)_{4} \mathrm{~N}-\mathrm{OH}$ & Biochem \\
\hline N-N, Dimethyl-dodecylamine (DMDDA) & $\mathrm{CH}_{3}\left(\mathrm{CH}_{2}\right)_{11} \mathrm{~N}\left(\mathrm{CH}_{3}\right)_{2}$ & Alfa Aesar \\
\hline Ethanol 96\% & $\mathrm{C}_{2} \mathrm{H}_{5} \mathrm{OH}$ & Biochem \\
\hline Janus Green B (Phenazinium) & $\mathrm{C}_{30} \mathrm{H}_{31} \mathrm{Cl}_{3} \mathrm{~N}_{6}$ & Alfa Aesar \\
\hline Orange II Sodium Salt & $\mathrm{C}_{16} \mathrm{H}_{12} \mathrm{~N}_{2} \mathrm{O}_{4} \mathrm{~S}-\mathrm{Na}$ & Sigma-Aldrich \\
\hline
\end{tabular}


The mixture is transferred to the Teflon reactor and placed in an oven set at $90^{\circ}$ to $100^{\circ} \mathrm{C}$. for 48 hours. Afterwards, one part of the surfactant is removed by Büchner filtration (filtration and washing with distilled water) and then placed in an oven for drying for one day at $100^{\circ} \mathrm{C}$. The material obtained has the following molar composition: $1 \quad \mathrm{SiO}_{2} ; 0.25$ CTAB; 0.2 TMAOH; $40 \mathrm{H}_{2} \mathrm{O}[6,7]$, is named: parent material « Si-MCM-41-P ».

Si-MCM-41, called material $\mathrm{P}$, is added to an emulsion consisting of water and N-NDimethyl-dodecylamine

(DMDDA) previously stirred for 5 minutes. This mixture is left under stirring for 30 minutes, then transferred to a Teflon reactor, which is placed in an oven set at $120^{\circ} \mathrm{C}$. for 3 days. The material obtained is filtered and washed several times with distilled water and then dried at ambient temperature: it is the "Si-MCM-41/A » amine material.

The selective extraction of the amine is carried out using a specific solvent which is the ethanol, using a soxhlet. The material obtained is the «Si-MCM-41/B » deaminated material.

The material Si-MCM-41 / P is calcined in $550^{\circ} \mathrm{C}$ during 7 hours giving rise to a material called «Si-MCM-41-P / $\mathbf{C}$ » parent / calcined material.

This step is carried out at $550^{\circ} \mathrm{C}$. for 7 hours with a heating rate of $1^{\circ} \mathrm{C} . / \mathrm{min}$, giving rise to a material called « Si-MCM41/C » calcined material.

\subsection{Characterization techniques:}

This characterization aims in a very thorough way, the study and the identification of the textural properties, structural and superficial, as well as the electrochemical properties of the surface of the obtained materials.
A combination of physicochemical (X-ray diffraction, BET, ATG, ATD and Zetrametry) and spectroscopic (FTIR) techniques is realized and their understandings could allow a good exploitation of these materials for specific applications.

\section{3- Results \& discussion :}

The X-ray diffraction at low angles is used to demonstrate the arrangement of the channels created by the micelles of surfactants.

Figure 1 shows the diffractograms measured between 0.5 and 6 degrees $(2 \theta)$ of the DMDDA-41A, DMDDA-41B and DMDDA-41C materials, respectively the DMDDA amine material, the previous material after deamination, and Calcined material. These four materials are compared to the MCM-41/P which is the parent material.

The main peak is observed at $2 \theta=2.28^{\circ}$ followed by three less intense peaks between $3.2<2 \theta<5.4^{\circ}$. This figure can be indexed in a crystallographic system and it is possible to attribute the peaks to diffractions on reticular planes indexed by Miller indices "hkl": the planes (100), (110), (200) and 210) as shown in the figure.

The diffractograms of the materials (amine, deaminated and calcined) have the same peaks as those observed on the purely silicic material MCM-41/P, which shows that the structure of the material is preserved after modifications made more than they all present an arrangement 2D hexagonal (p6mm) of the pores (Figure 2). [8] 


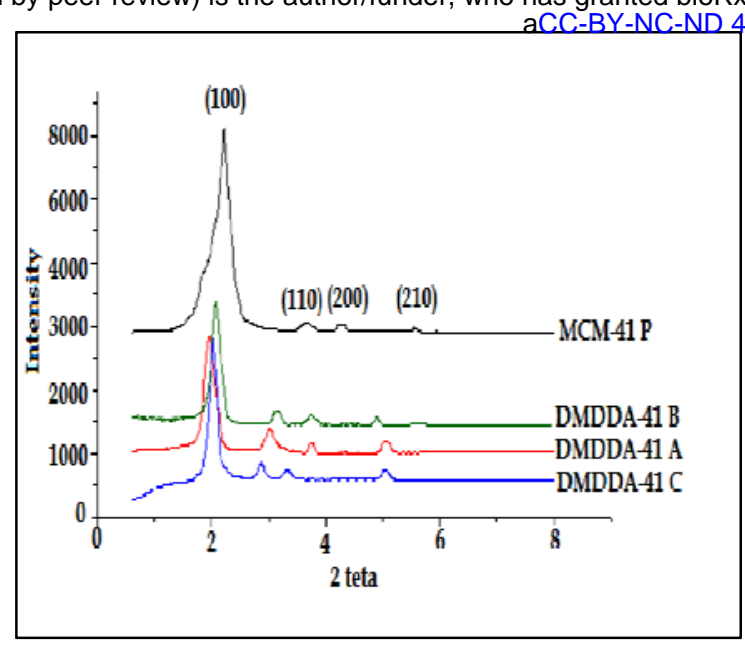

Figure 1: Si-MCM-41 diffraction pattern (parent, amine, deaminated, calcined)

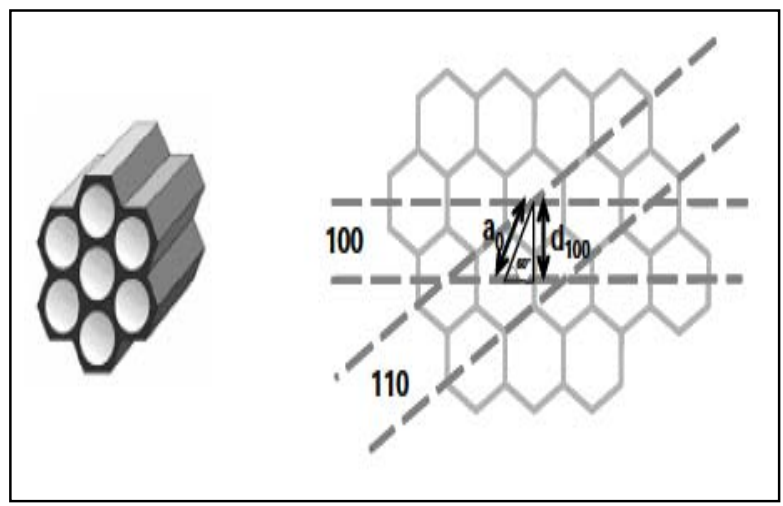

Figure 2: Diagram of the pore arrangement.

A peak slippage (MCM-41P and DMDDA41B) is observed at higher diffraction angles $[9,10,13]$, which results in smaller inter-reticular distances as shown in the table.

Table 2 summarizes the structural data derived from the diffractograms for all materials prepared from MCM-41.

Table 2: X-ray diffraction data of MCM-41P and modified materials.

\begin{tabular}{|l|c|c|c|}
\hline \multicolumn{1}{|c|}{ Material } & $2 \theta$ & $\begin{array}{c}\mathrm{d}_{100} \\
(\mathrm{~nm})\end{array}$ & $\begin{array}{c}\mathrm{a}_{0} \\
(\mathrm{~nm})\end{array}$ \\
\hline MCM-41 P & 2,28 & 3,87 & 4,47 \\
\hline DMDDA-41A & 1,97 & 4,39 & 5,07 \\
\hline DMDDA-41B & 2,10 & 4,21 & 4,86 \\
\hline DMDDA-41C & 2,02 & 4,48 & 5,17 \\
\hline
\end{tabular}

The adsorption-desorption isotherms of the parent MCM-41 and the prepared materials are shown in figure 3 . These are type IV according to the IUPAC classification and Hysteresis $\mathrm{H} 1$ which is representative of structured mesopores. [11, 12, 13, 30]

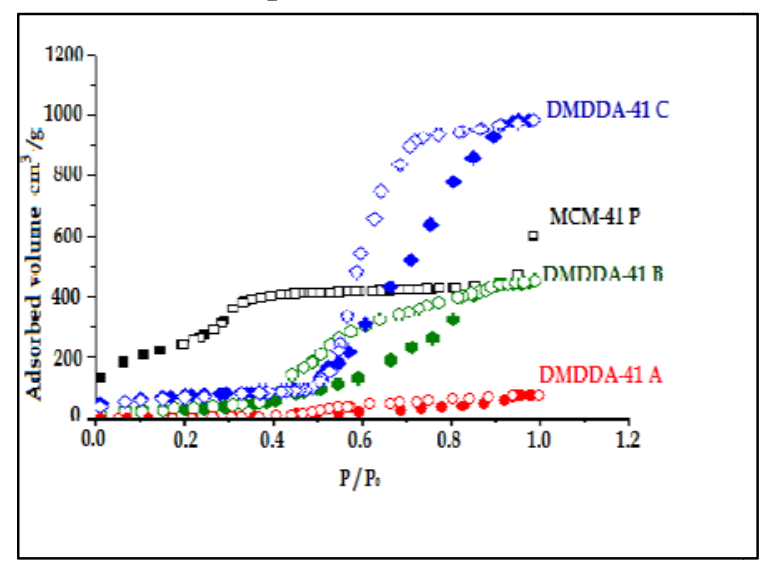

Figure 3: Isotherm of adsorption / desorption of Si-MCM-41

For the MCM-41P, an increasing slope is observed for low relative pressures $0<(\mathrm{P} /$ P0) $<0.3$ corresponding to a monolayer filling of the surface [10], followed by a steep slope of the curve for the Relative pressures between $0.3<(\mathrm{P} / \mathrm{P} 0)<0.8$; Due to the capillary condensation of the nitrogen inside the mesopores. And towards $(\mathrm{P} / \mathrm{P} 0)>0.8$ there is a multilayer adsorption at the surface of the parent material.

For the DMDDA-41A material, at relative pressures $\mathrm{P} / \mathrm{P} 0<0.4$, a monolayer filling of the surface is obtained and for $\mathrm{P} / \mathrm{P} 0>$ 0.5 there appears a plateau of a multilayer adsorption. The same observations noted for the parent material are noted for DMDDA-41B and DMDDA-41C. For both materials, the relative low pressure plateau is almost the same ( $\mathrm{P} / \mathrm{P} 0<0.5)$, but the distinction is in the slopes of the following plateau: steep slope to the calcined material (up to 1 in $\mathrm{P} / \mathrm{P} 0$ ) in contrast to the deaminated material $(0.5<\mathrm{P} / \mathrm{P} 0<0.8)$.

In the case of these two materials, the desorption does not follow the adsorption creating an $\mathrm{H} 1$ type hysteresis which 
abruptly closes at $\mathrm{P} / \mathrm{P} 0=0.5$ for the material (DMDDA-41C) and at $\mathrm{P} / \mathrm{P} 0=$ 0.4 For the DMDDA-41B material, this suggests a phenomenon of capillary condensation in the mesopores. The width of the hysteresis increases (DMDDA-41C) indicating the pore size distribution is much wider in the calcined material (Figure 4) [13, 23, 24].

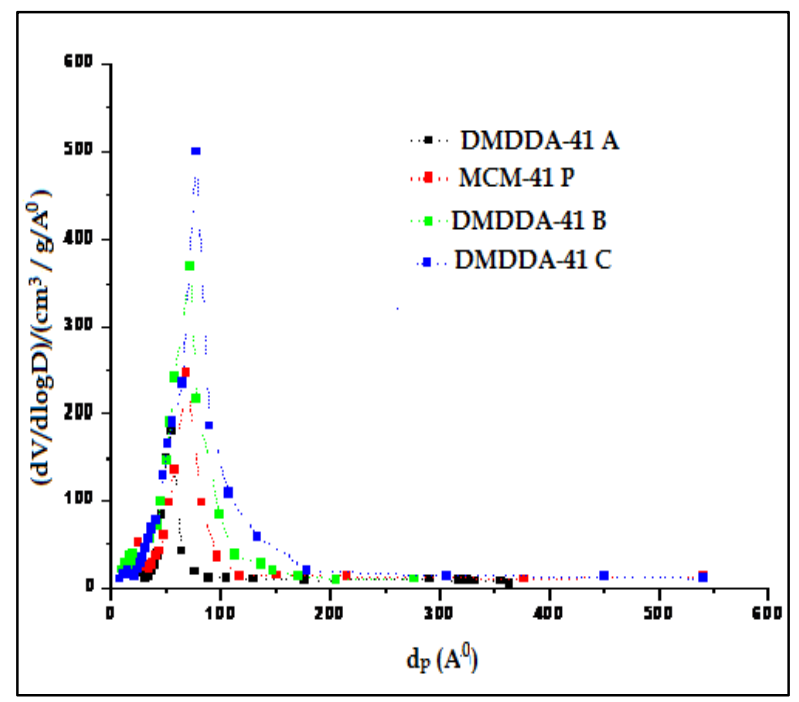

Figure 4 : Distribution of pore diameters of materials MCM-41

The pore diameter distributions shown in figure 4 show that the materials prepared have different pore diameters. The traces are all centered indicating a better uniformity of the pore diameter. As the pore diameter distribution widens, this indicates the formation of a less homogeneous network of pores.

The analysis of these data makes it possible to observe great differences in the parameters of the four materials. The results of surface area, pore diameter and pore volume decrease remarkably for the amine material (the surface and part of the pore volume are occupied by the amines), in contrast to MCM-41P.
Afterwards, deamination increases these parameters again, while calcination leads to higher values.

Table 3: Comparison of nitrogen adsorptiondesorption data at $77^{\circ} \mathrm{K}$ of MCM-41P and modified materials.

\begin{tabular}{|l|c|c|c|}
\hline \multicolumn{1}{|c|}{ Material } & $\begin{array}{c}\mathbf{S}_{\mathbf{B E T}} \\
\left(\mathbf{m}^{\mathbf{2}} / \mathbf{g}\right)\end{array}$ & $\begin{array}{c}\mathbf{d}_{\mathbf{P}} \\
(\mathbf{n m})\end{array}$ & $\begin{array}{c}\mathbf{V}_{\mathbf{p}} \\
\left(\mathbf{c m}^{\mathbf{3}} / \mathbf{g}\right)\end{array}$ \\
\hline MCM-41 P & 1147 & 3,22 & 0,85 \\
\hline DMDDA-41A & 74 & - & 0,28 \\
\hline DMDDA-41B & 387 & 5,84 & 1,07 \\
\hline DMDDA-41C & 1246 & 10,6 & 1,86 \\
\hline
\end{tabular}

The IRTF spectra of the MCM-41 materials prepared are shown in figure 5 where it is found that some vibration bands are present in the materials: they are similar to that of amorphous silica.

The broad band between $3450 \mathrm{~cm}^{-1}$, characteristic of elongation of the bond $(\mathrm{O}$ $\mathrm{H})$, water and silanol groups of the surface. Another vibrating band $(\mathrm{O}-\mathrm{H})$ noted around $1650 \mathrm{~cm}^{-1}$ testifying to the water present in the materials. At $1100 \mathrm{~cm}^{-1}$, an asymmetric (O-Si-O) elongation junction of the $\mathrm{SiO}_{4}$ tetrahedral entities was observed, whereas about 950 and $750 \mathrm{~cm}^{-1}$, two bends characteristic of the asymmetric elongation of the ( $\mathrm{Si}-\mathrm{O}$ ) bond of these same entities present. We note also the presence of a band characterizing the deformation of the angle (O-Si-O) of the tetrahedral entities $\mathrm{SiO}_{4}$, this towards $450 \mathrm{~cm}^{-1} \cdot[14,16,21]$

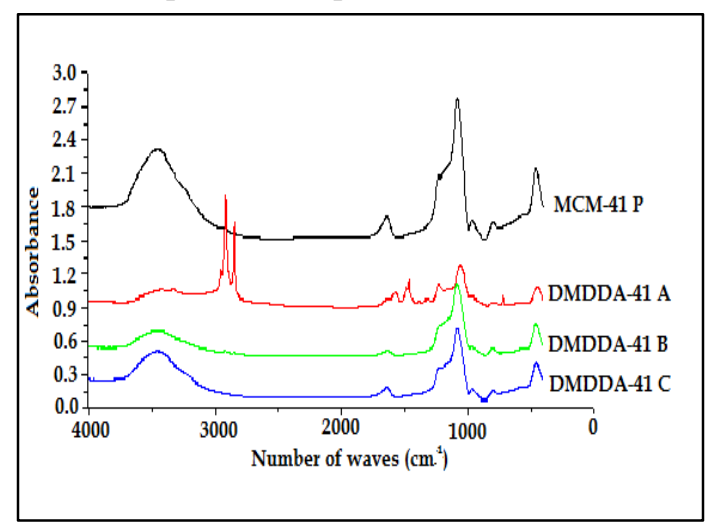

Figure 5: IRTF Spectrum of Si-MCM-41 
In addition to these bands, the amine material (DMDDA-41A) records two new bands characteristic of the amine groups, the first located at $2900 \mathrm{~cm}-1$ characterizes the vibrations $(\mathrm{CN})$, while the second one is observed at $1480 \mathrm{~cm}-1$ Resulting from the deformation vibration of the bonds (NH). [14, 18, 22]

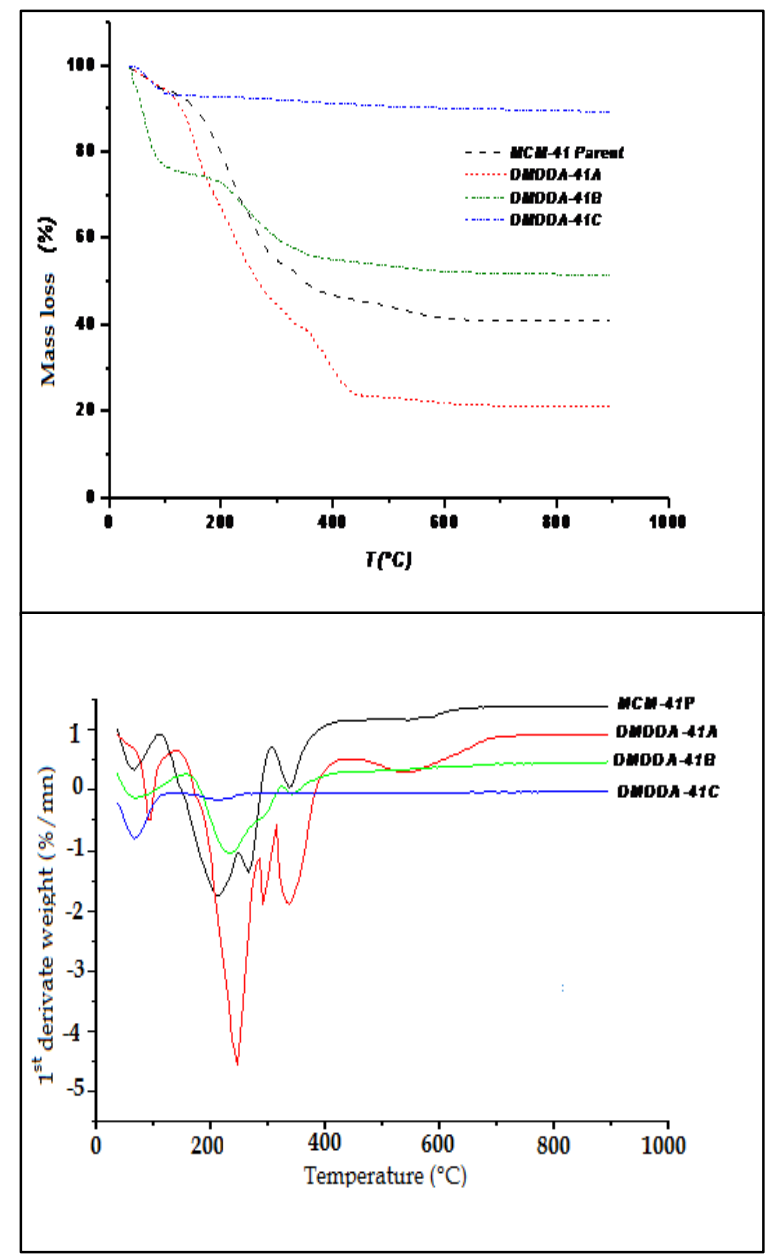

Figure 6 : Thermogravimetric analysis A- (TGA) \& Differential thermal analysis B-(DTA) of the different materials

The ATG / ATD thermogravimetric analysis of all the materials is represented by two figure6 ( $\mathrm{A}$ and $\mathrm{B}$ ), from which there are three different zones of mass variation as a function of temperature.

The $1^{\text {st }}$ zone at low temperature $(25<\mathrm{T}$ $<160^{\circ} \mathrm{C}$.) corresponds to dehydration of the water physisorbed on the surface of the material. The $2^{\text {nd }}$ intermediate zone $(160<\mathrm{T}$ $<600^{\circ} \mathrm{C}$ ) corresponds to the decomposition and volatilization of the organic compounds (surfactants and amine) in strong interaction with the surface of the material (covalent and electrostatic bonds) The last high temperature zone $(600<\mathrm{T}$ $<900^{\circ} \mathrm{C}$ ):assigned to silica dehydroxylation phenomena (condensation of the remaining silanols causing the elimination of water molecules) [8, 10, 15, 17, 24].

Table 4: Thermogravimetric (TGA) and

Thermal Differential (ATD) data for the different materials

\begin{tabular}{|c|c|c|c|}
\hline \multirow{2}{*}{ Sample } & \multicolumn{3}{|c|}{ Mass loss (\%) } \\
\cline { 2 - 4 } & $\begin{array}{c}\mathbf{1}^{\text {st }} \\
\text { Zone }\end{array}$ & $\begin{array}{c}\mathbf{2}^{\text {nd }} \\
\text { Zone }\end{array}$ & $\begin{array}{c}\mathbf{3}^{\text {rd }} \\
\text { Zone }\end{array}$ \\
\hline MCM-41 P & 8,23 & 48,41 & 1,43 \\
\hline DMDDA -41 A & 3,51 & 70,67 & 3,59 \\
\hline DMDDA -41 B & 15,67 & 45,97 & 1,63 \\
\hline DMDDA -41 C & 7,02 & 2,76 & 0,87 \\
\hline
\end{tabular}

Figure 7 shows the curves $\zeta=\mathrm{f}(\mathrm{pH})$ of the various MCM-41 materials in the $\mathrm{pH}$ range from 2 to 11. Each point $\zeta=\mathrm{f}(\mathrm{pH})$ is the mean of the results obtained on the measurements of the electrophoretic mobility of 100 to $500 \mathrm{p}$ at a zeta potential of between 5 and $8 \mathrm{mV}$. The $\mathrm{pH}$ for which the zeta potential is zero (no displacement of the particles under the effect of the electric field) is called the isoelectric point (PIE). [19, 20]

Parent material MCM-41 has positive potentials between $2<\mathrm{pH}<5$ and negative potentials from $\mathrm{pH}=5.5$. For the DMDDA-41 A, it has positive potentials between $2<\mathrm{pH} \quad<8.5$ and negative potentials from $\mathrm{pH}=8.5$. Then, DMDDA$41 \mathrm{~B}$ has positive potentials between $2<\mathrm{pH}$ $<6$ and negative potentials from $\mathrm{pH}=6.1$. 
And for the calcined material, it has positive potentials between $2<\mathrm{pH}<3.6$ and negative potentials from $\mathrm{pH}=3.6[16$, $18,23,24]$.

Table 5: Values of the isoelectric points of the different materials.

\begin{tabular}{|l|c|}
\hline Material & PIE (isoelectric point) \\
\hline MCM-41 P & 5,6 \\
\hline DMDDA -41 A & 8,7 \\
\hline DMDDA -41 B & 5,8 \\
\hline DMDDA -41 C & 3,6 \\
\hline
\end{tabular}

\section{4- Application to adsorption}

Our study consists in developing techniques for low - cost water discolouration while recovering industrial waste: Discoloration of synthetic solutions: Orange 2 sodium with anionic character [25] and Janus Green $\boldsymbol{B}$ with a cationic character. Their characteristic data are summarized in the following table.

Table 6 : Physico-chimical characteristics of the two dyes

\begin{tabular}{|c|c|c|}
\hline & Janus Green B & Orange II Sodium Salt \\
\hline $\begin{array}{l}\text { IUPAC } \\
\text { name }\end{array}$ & $\begin{array}{l}\text { 3-Diethylamino-7-(4- } \\
\text { dimethylaminophenylazo)-5- } \\
\text { phenylphenazinium chloride }\end{array}$ & $\begin{array}{l}\text { 4-(2-Hydroxy-1- } \\
\text { naphthylazo)benzenesulfonic acid } \\
\text { sodium salt }\end{array}$ \\
\hline Formula & $\mathrm{C}_{30} \mathrm{H}_{31} \mathrm{CLN}_{6}$ & $\mathrm{C}_{16} \mathrm{H}_{12} \mathrm{~N}_{2} \mathrm{O}_{4} \mathrm{~S}-\mathrm{Na}$ \\
\hline $\begin{array}{l}\text { Developed } \\
\text { formula }\end{array}$ & & \\
\hline Solubility & Soluble & Soluble \\
\hline Stability & Stable in NTPs & Stable in NTPs \\
\hline Appearance & Blue-green powder & Orange powder \\
\hline $\begin{array}{l}\text { Molecular } \\
\text { weight }\end{array}$ & $511.06 \mathrm{~g} / \mathrm{mol}$ & $350.32 \mathrm{~g} / \mathrm{mol}$ \\
\hline Absorbance & $\square_{\max 1}=395 \mathrm{~nm}, \square_{\max 2}=660 \mathrm{~nm}$ & $\square_{\max }=483 \mathrm{~nm}$ \\
\hline
\end{tabular}

First, a study of their UV-visible spectra at wavelengths between 200 and $800 \mathrm{~nm}$ was carried out to determine the wavelengths which correspond to the maximum adsorption and to establish the calibration curves. Thus the $483 \mathrm{~nm}, 618 \mathrm{~nm}$ waves were respectively for the Orange II Sodium Salt and the Janus Green B. We then studied the influence of different physicochemical parameters on the coloring interactions - synthesized materials (agitation time, Initial dye concentration, adsorption capacity test).

It is noted that in each experiment, the tests were carried out in flasks covered with aluminum foil, in order to avoid degradation of the pollutant in the presence of light.

The study of the adsorption of the two dyes on the different materials is represented in the histograms of the quantities adsorbed by the various materials synthesized (figure 8) 
We note that the two materials "parent and amine" possess the greatest adsorption capacity of the anionic dye "Orange II". On the other hand, the two materials, namely the calcined parent and the calcined modifier, possess the highest adsorption rates of the cationic dye "Janus GB". The DMDDA-41 B exhibits in both dyes an average adsorption

This difference in the adsorption rates of the different materials is due to two reasons:

1. Differences in the physico-chemical properties of materials (porosity, $\mathrm{S}_{\mathrm{BET}}, \ldots$ )

2. Differences in surface loads of different materials.

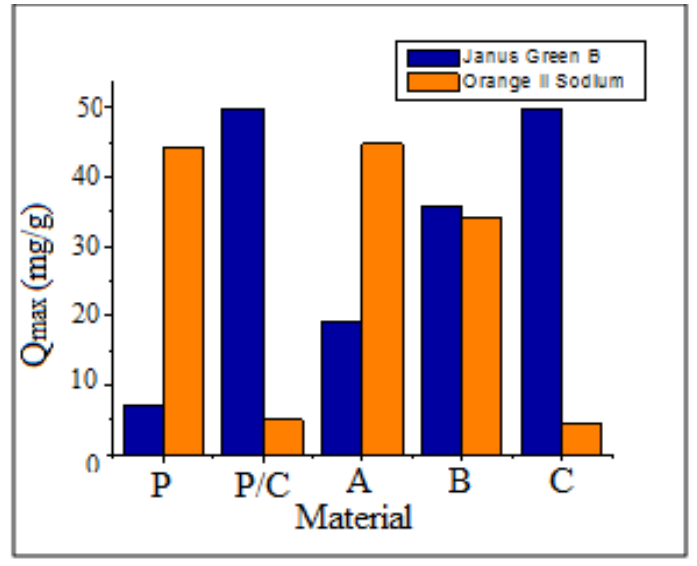

Figure 8 : The retention quantities of the two dyes by the various materials MCM-41

The parent material has only the silanol $(\mathrm{Si}-\mathrm{OH})$ groups and quaternary ammonium groups of the surfactant, which explains its ability to fix the anionic dye more than the cationic. Moreover, the amine material has, in addition to the preceding groups [27], amine groups (NH.sub.2): the charge of their surface is more positive at the maximum capacity for fixing the Orange II.

For calcined materials (DMDDA-41 P / C and DMDDA-41 C), they have only the silanol groups $(\mathrm{Si}-\mathrm{OH})$ which make the charge of their surface more negative, which makes their adsorption capacity of the Janus GB maximum.

Table 7: Retention quantities of Orange II

Sodium and Janus Green B by the different materials

\begin{tabular}{|l|c|c|c|c|c|}
\hline $\begin{array}{c}\text { Material } \\
\text { MCM-41 }\end{array}$ & P & P/C & A & B & C \\
\hline $\begin{array}{l}\text { Q }_{\max }(\mathrm{mg} / \mathrm{g}) \\
\text { « Orange II } \\
\text { Sodium » }\end{array}$ & $\mathbf{4 4 . 3 4}$ & $\mathbf{4 . 9 6}$ & $\mathbf{4 4 . 8 0}$ & $\mathbf{3 3 . 9 8}$ & $\mathbf{4 . 2 8}$ \\
\hline $\begin{array}{l}\mathrm{Q}_{\max }(\mathrm{mg} / \mathrm{g}) \\
\text { « Janus GB }\end{array}$ & $\mathbf{7 . 0 5}$ & $\mathbf{4 9 . 8 0}$ & $\mathbf{1 9 . 1 3}$ & $\mathbf{3 5 . 7 0}$ & $\mathbf{4 9 . 9 0}$ \\
\hline
\end{tabular}
out on the amine and calcined matrix, since they possess, respectively, the greatest adsorption capacity of the anionic and cationic micropollutant. The results are presented by the evolution of the adsorbed quantities of the two dyes by the two materials $(\mathrm{Qe})$ as a function of the contact time $(\mathrm{t})$ in FIG. 9 (A and B).

In the two figures (A and $\mathrm{B}$ ), two distinct phases of adsorption are observed: the first between 0 and 40 minutes which is rapid and takes place at the sites accessible on the surface, followed by a slow evolution of the adsorbed amount before to reach the second phase, which is in the form of an equilibrium plateau, due to the diffusion inside the pores of the material. The balance is reached after 60 minutes.

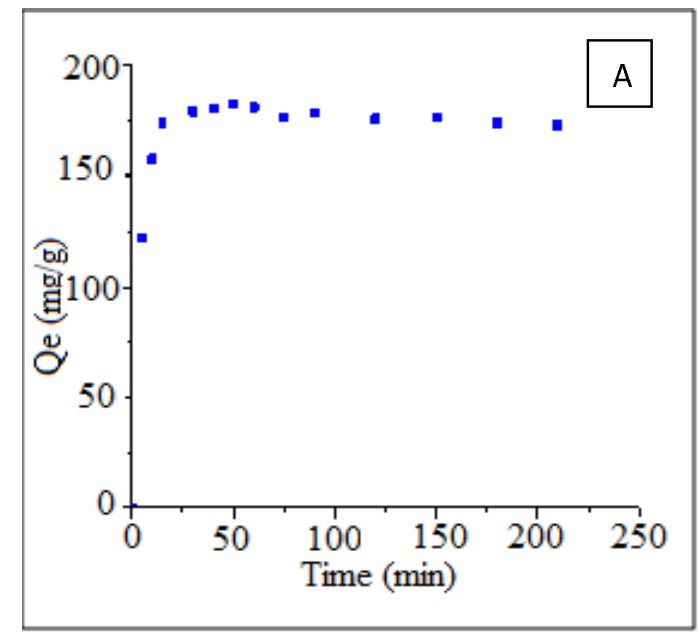




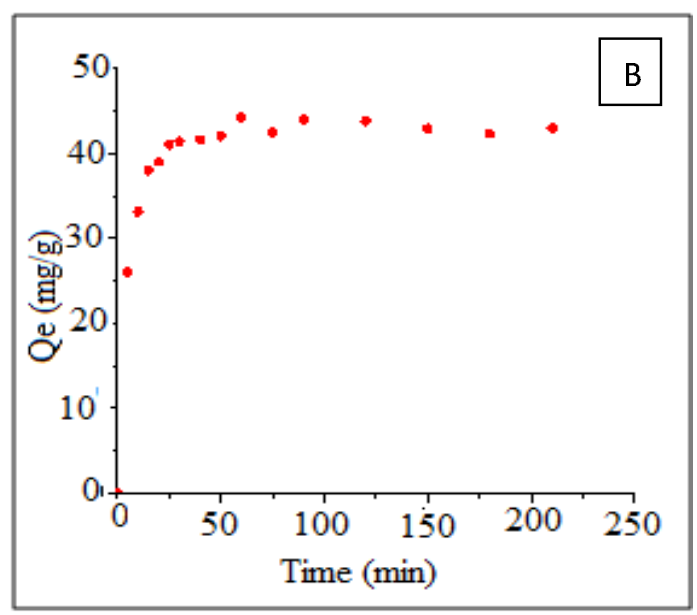

Figure 9 : Kinetics of elimination of Janus Green B by DMDDA-41C (A) and Orange II Sodium by DMDDA-41A (B)

Fiigures 10 and 11 represent the linearization of the two kinetic models for the two organic pollutants and Table 8 groups together the values of the parameters of the two kinetic models applied to their elimination.
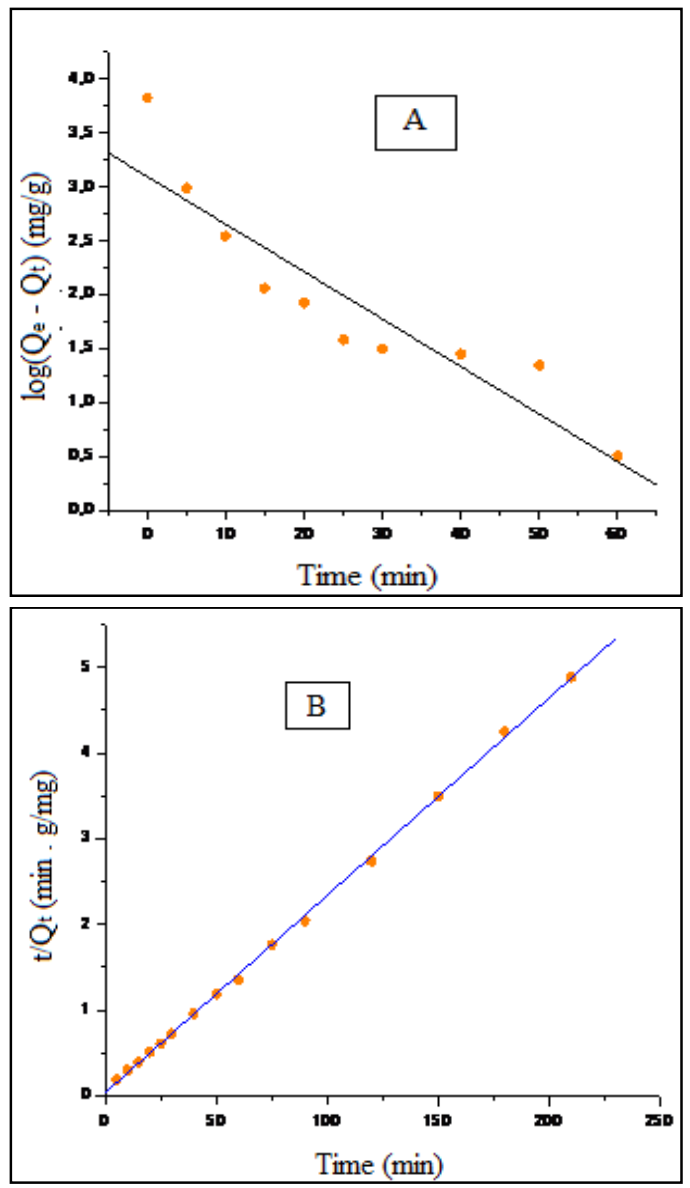

Figure 10 : Application of the pseudo-first order (A) and pseudo-second order (B) model to the elimination of Orange II Sodium by DMDDA-

41A
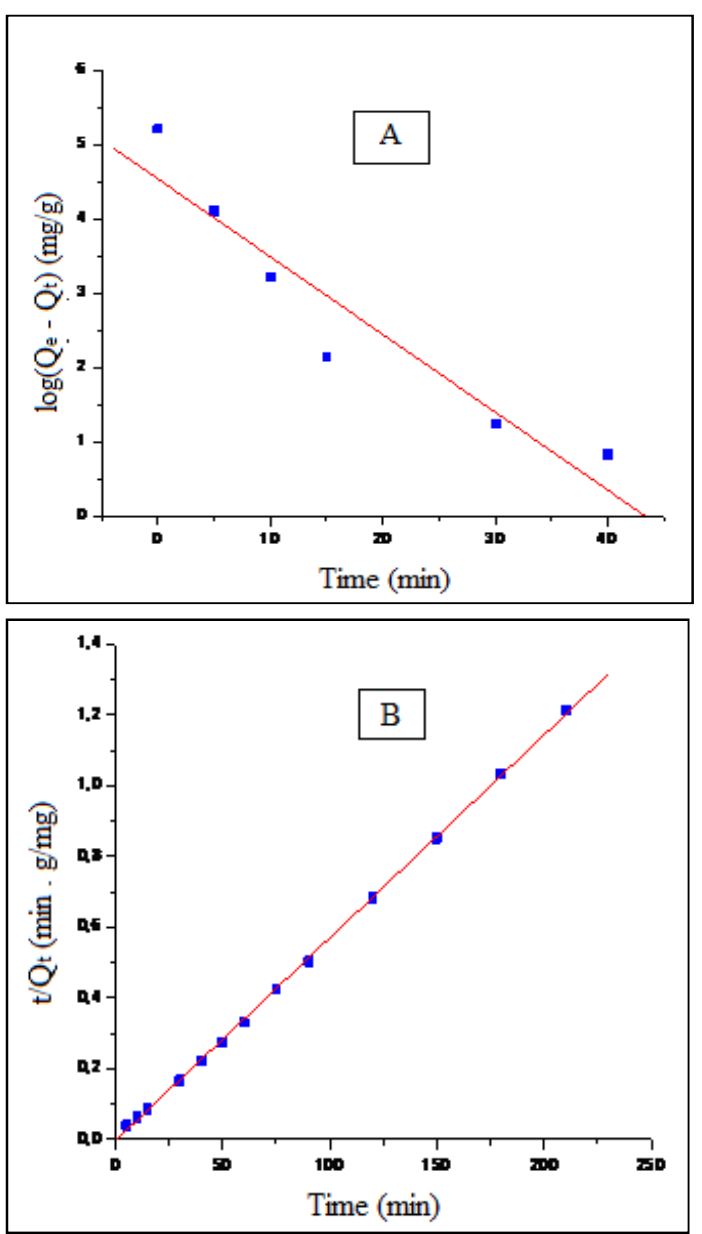

Figure 11: Application of the pseudo-first order (A) and pseudo-second order (B) model to the elimination of Janus Green B by the DMDDA-

41C

According to the values of the kinetic constants, the kinetics of the dyes follows the pseudo second order model $\left(\mathrm{R}^{2}=\right.$ 0.999), rather than that of the pseudo-first. 
Table 8: Parameters of the two kinetic models applied to the removal of the two organic pollutants "Janus Green B \& Orange II

Sodium"

\begin{tabular}{|c|c|c|c|c|c|c|}
\hline \multirow[b]{2}{*}{ Material } & \multicolumn{3}{|c|}{$\begin{array}{l}\text { Pseudo } \\
\text { First ordre }\end{array}$} & \multicolumn{3}{|c|}{$\begin{array}{c}\text { Pseudo } \\
\text { Second ordre }\end{array}$} \\
\hline & $\begin{array}{c}\mathrm{K}_{1} \\
\left(\mathbf{m i n}^{-}\right. \\
\left.\mathbf{1}^{\prime}\right)\end{array}$ & $\begin{array}{c}\text { Qe } \\
(\mathbf{m g} / \\
\text { g) }\end{array}$ & $\mathbf{R}^{2}$ & $\begin{array}{c}\mathbf{K}_{2} \\
\left(\mathbf{g} \cdot \mathrm{min}^{-}\right. \\
\left.{ }^{1} \cdot \mathrm{mg}^{-1}\right)\end{array}$ & $\begin{array}{c}\text { Qe } \\
(\mathbf{m g} / \mathbf{g})\end{array}$ & $\mathbf{R}^{2}$ \\
\hline $\begin{array}{c}\text { DMDDA } \\
-41 \mathrm{C}\end{array}$ & 0.013 & $\begin{array}{c}14.1 \\
3\end{array}$ & 0.78 & 0.010 & 174.52 & 0.99 \\
\hline $\begin{array}{c}\text { DMDDA } \\
-41 \mathrm{~A}\end{array}$ & 0.019 & 8.76 & 0.84 & 0.014 & 43.42 & 0.99 \\
\hline
\end{tabular}

The plots of the curves: $\mathrm{Qt}=\mathrm{f}(\mathrm{t} 1 / 2)$, of the intraparticle diffusion (FIG. 12), are not linear and we notice three distinct sections associated with three successive steps:

- A first linear portion of the first step corresponds to a rapid diffusion in the boundary layer of the molecules of the solute, the adsorbate migrating from the solution to the external surface of the adsorbent.

- The second section, the curvature, is attributed to the intraparticle diffusion, which determines the rate of control of the adsorption mechanism.

- The plateau corresponds to a state of equilibrium: the intra-particle diffusion slows down, leading to a maximum of adsorption and a very low concentration of adsorbate in the solute [29]

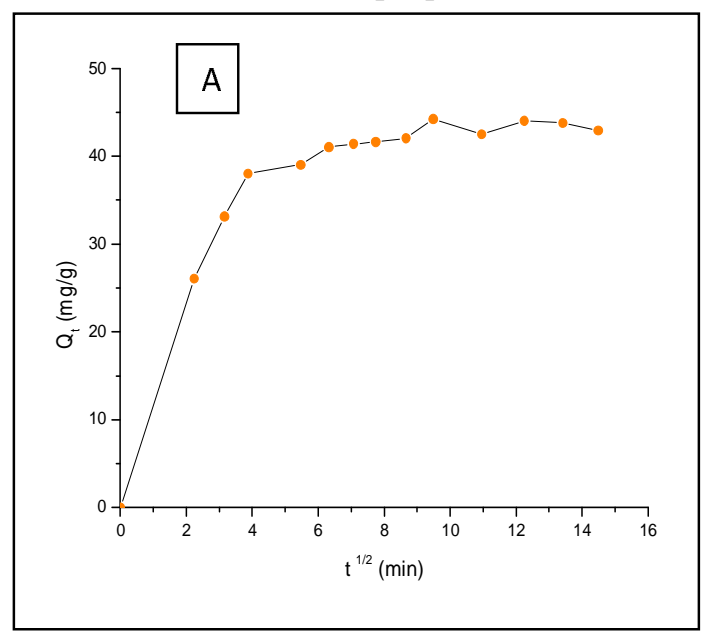

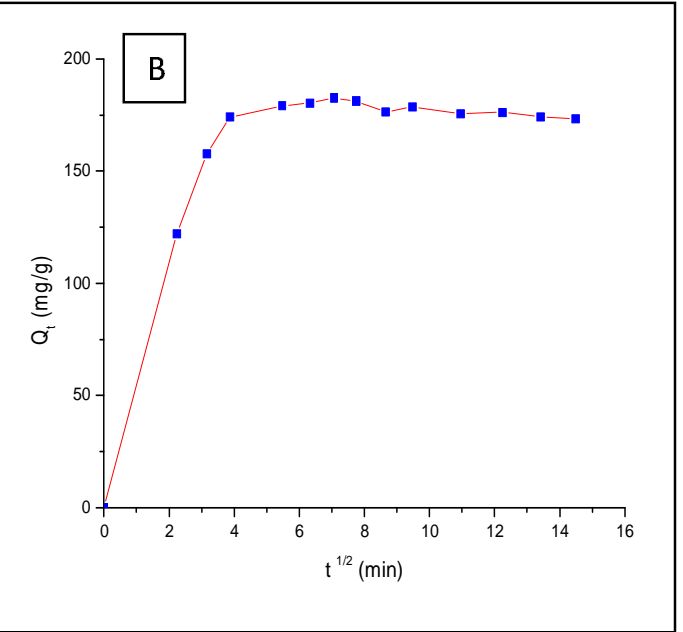

Figure 12: Intraparticular diffusion of Orange II Sodium (A) and Janus Green B (B)

Table 9: Kinetic parameters of the intraparticular diffusion of the two dyes "Janus Green B \& Orange II Sodium"

\begin{tabular}{|c|c|c|c|c|}
\hline \multirow{2}{*}{ Material } & \multicolumn{4}{|c|}{ Intraparticular diffusion } \\
\cline { 2 - 5 } & $\begin{array}{c}\mathbf{Q}_{\mathbf{e}(\mathbf{e x p})} \\
(\mathbf{m g} / \mathbf{g})\end{array}$ & $\begin{array}{c}\mathbf{K}_{\text {id }} \\
\left(\mathbf{m g} / \mathbf{g} \cdot \mathbf{m i n}^{\mathbf{1} / 2}\right)\end{array}$ & $\begin{array}{c}\mathbf{I} \\
(\mathbf{m g} / \mathbf{g})\end{array}$ & $\mathbf{R}^{\mathbf{2}}$ \\
\hline $\begin{array}{c}\text { DMDDA- } \\
\text { 41A }\end{array}$ & $\mathbf{4 4 . 2 4}$ & $\mathbf{2 . 0 0 9}$ & $\mathbf{2 2 . 0 4 4}$ & $\mathbf{0 . 7 4 0}$ \\
\hline $\begin{array}{c}\text { DMDDA- } \\
\text { 41C }\end{array}$ & $\mathbf{1 8 2 . 6 4}$ & $\mathbf{6 . 7 4 8}$ & $\mathbf{1 0 8 . 7 4 1}$ & $\mathbf{0 . 6 0 5}$ \\
\hline
\end{tabular}

The adsorption isotherms of the anionic and cationic dye at $25^{\circ} \mathrm{C}$. by the materials DMDDA-41A and DMDDA-41C are shown in figure 13.

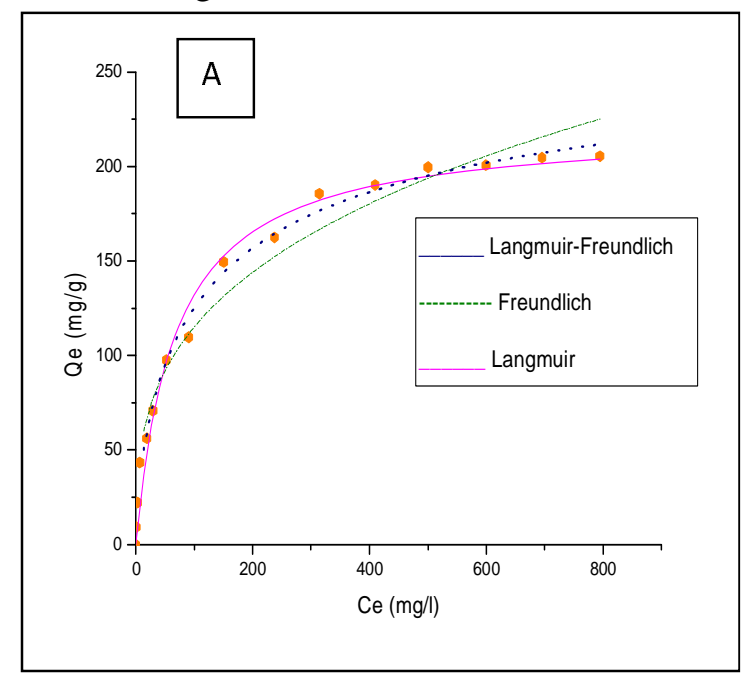




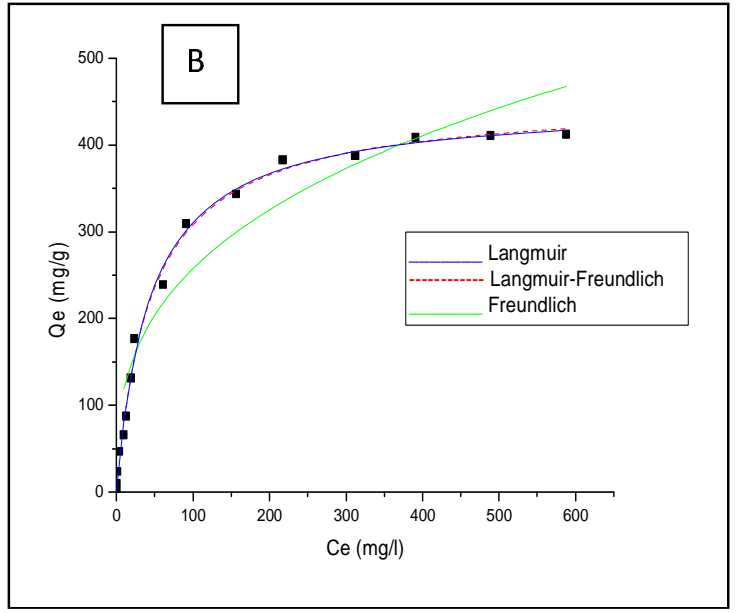

Figure 13: Adsorption isotherms of Orange II Sodium by DMDDA-41A (A) and Janus Green B by DMDDA-41C (B)

Using the classification of adsorption isotherms, those obtained are of type L "Langmuir". This type is characterized by a decreasing slope as the equilibrium concentration increases, due to the decrease in the number of adsorption sites, following the gradual covering of the surface of the various materials. In this type of adsorption, there is no interaction between the adsorbed molecules: this is a physisorption [28]. The representation of the adsorption isotherms is markedly better by the Sips model in the two dyes $\left(\boldsymbol{R}^{2}>\right.$ 0.994) and especially by the Langmuir model in the fixation of the cationic pollutant Janus Green B $\left(\boldsymbol{R}^{2}>\mathbf{0 . 9 9 5}\right)$.

The study of the linearizations of the Langmuir, Freundlich and Sips models (Langmuir-Freundlich), defined above, gives the various parameters of the three models that we have gathered in Table 10.

Table 10: Parameters of the adsorption isotherms applied to the removal of the two organic pollutants "Janus Green B \& Orange II Sodium"

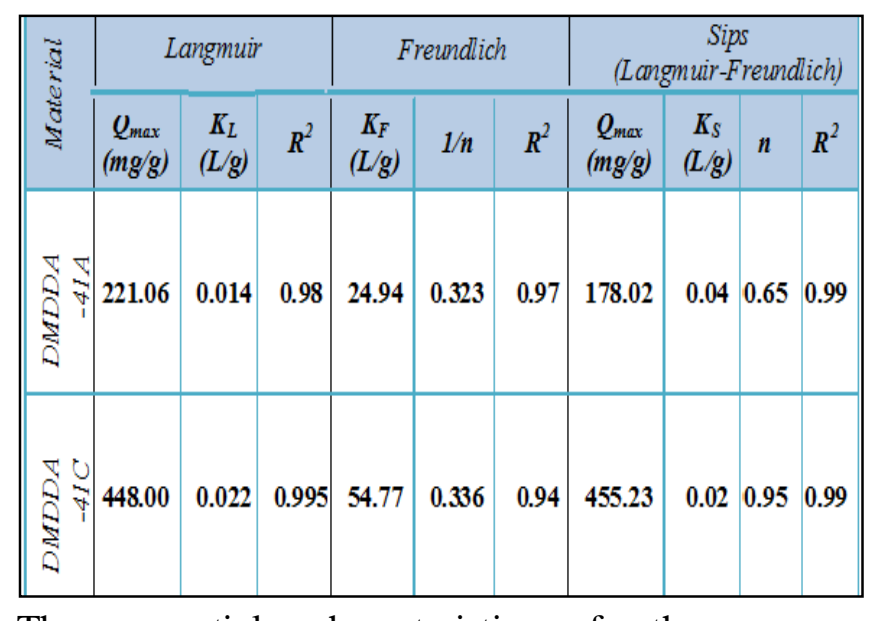

The essential characteristic of the Langmuir isotherm can be expressed as a function of a dimensionless constant separation factor which is given by the following expression:

$$
\mathbf{R}_{\mathrm{L}}=\left[1 /\left(1+\mathrm{K}_{\mathrm{L}} * \mathbf{C}_{0}\right)\right]
$$

The evolution of the separation factors as a function of the initial concentration at $25^{\circ}$ $\mathrm{C}$ is shown in figure 14. For both materials and the two dyes, the values of this factor are between $\boldsymbol{0}<\boldsymbol{R} \boldsymbol{L}<\boldsymbol{1}$ : This shows that the adsorption of the two dyes by the different materials is a favorable process, and the $1 / \boldsymbol{n}$ values indicate a favorable adsorption especially for the low concentrations [26, 27].

\section{Conclusion :}

The main objective of this work was to synthesize the MCM-41 material from optimized protocols. It was then possible to increase the size of the pores and the specific surface area of the parent material by the post-synthesis incorporation of blowing agents (long chain carbon amines DMDDA).

The results of X-ray diffraction characterization of the different matrices show that for the five types of morphology the diffractogram obtained has three indexable peaks in (100), (110) and (200) in hexagonal symmetry (space group 
P6mm) . For amine and calcined materials, the presence of indexable peaks in hexagonal network always shows that the structure is always preserved after the postsynthesis addition of the amine emulsion or by a high treatment temperature.

The BET analysis of the materials shows a remarkable decrease in pore diameter and the specific surface area for each of the amines and deaminates, unlike the MCM$41 \mathrm{C}$ which has the highest surface area and pore diameter.

The thermogravimetric analysis shows a thermal stability of the materials with a greater loss of mass for the amine material, which again confirms the incorporation of the amines inside the pores.

The results of the infrared spectra show the incorporation of the $\mathrm{NH}_{2}$ groups and for those of the zeta-metry show that the surface charge differs from one material to another, which suggests their applications in the adsorption domain of the various micropollutants .

The results obtained during the adsorption study show the effectiveness of these materials, in particular "the amine material" and "the calcined material" for decolorizing aqueous media contaminated with organic dyes. The kinetic studies and the adsorption isotherms were carried out to clarify the method of fixing each of the two dyes on the two materials tested. The experiments showed that the amine material had a maximum capacity for fixing Orange II (221.06 mg / g) (anionic dye), whereas for a maximum adsorption capacity (455.23 mg / g ) of Janus GB (cationic dye), the calcined material is more efficient.

The second order kinetic model applies well in the case of the adsorbent / adsorbate systems studied $\left(\mathrm{R}^{2}=0.999\right)$. The adsorption isotherms of the two dyes by the two adsorbents are satisfactorily described by the Langmuir model in the case of fixation of the cationic pollutant $\left(\mathrm{R}^{2}=0.995\right)$. However, for the adsorption of the anionic pollutant, the Sips model is more favorable $\left(\mathrm{R}^{2}=0.994\right)$.

The RL separation factor values for the Langmuir model of the two dyes is between $(0<\mathrm{RL}<1)$ : this shows that the adsorption in this case is a favorable process.

The adsorption of the two micropollutants (Janus Green B and Orange II Sodium) is favored at low concentrations $(1 / \mathrm{n}<1)$.

\section{References}

[1] L. B. McCusker; F. Liebau ; G. Engelhardt : «Micoporous and Mesoporous Materials». Pure Appl. Chem. 2001, 73, 381-394.

[2] F. Lebiau: " Adsorption, Surface Area and Porosity ». Micopor. Mesopor. Mater, 2003, 58, 1572.

[3] T. Sano, K. Doi, H. Hagimoto, Z. Wang, T. Uozumi and K. Soga, Chem. Commun., 1999, 733. [4] A. Benhamou, J.P. Basly,M. Baudu, Z. Derriche , R. Hamacha : "Aminofunctionalized MCM-41 and MCM-48 for the removal of chromate and arsenate » Journal of Colloid and Interface Science ; 404 (2013) 135-139.

[5] S. Barclay, C. Buckley, «Waste minimization guide for the textile industry, a step towards cleaner production». The pollution research group, Water research Commission, 2000,I.

[6] A. Benhamou: Thesis «Use of Functionalized Mesoporous Materials in Water Treatment», University of Science and Technology of Oran Mohamed Boudiaf, 2010, p 56-58.

[7] N.VAAND et S. RAJPUT : « Mesoporous Material, MCM41: A new drug carrier ». Asian Journal of Pharmaceutical and Clinical Research, Vol. 4, Issue 2, 2011, ISSN - 0974-2441.

[8] N.Crowther: Thesis: "Catalysts based on Platinum Complexes Incorporated in the Walls of Periodic Mesoporous Silicas; Reactivity in Hydrogenation ". Thesis of Doctorate, Ecole Normale Supérieure of Lyon, France, 2007, 80-86.

[9] A. M.Showkat; Y. P. Zhang; M. S. Kim; A. Lyenger; G. Karla; Ra. Reddy; K; P. Lee;" Analysis of Heavy Metal Toxic Ions by Adsorption onto Amino-functionalized ordered Mesporous Silica”. Bull. Kor. Chem. Soc. 2007, 28, 1985-1992. 
[10] S. ABRY: Thesis: "Surface molecular engineering applied to the design of heterogeneous bio-inspired catalysts". Thesis of Doctorate, Ecole Normale Supérieure of Lyon, France, 2007, p 109120.

[11] C.T. Kresge; M.E.Leonowcz; J.W Roth; J.C. Vartuli; J.S. Beck;" Ordered mesoporous molecular sieves synthesized by a liquid-crystal template mechanism”.Narture, 1992, 359,710-712.

[12] Dada, A.O1, Olalekan, A.P, Olatunya, A.M, DADA, O : «Langmuir, Freundlich, Temkin and Dubinin-Radushkevich Isotherms Studies of Equilibrium Sorption of $\mathrm{Zn} 2+$ Unto Phosphoric Acid Modified Rice Husk ». IOSR Journal of Applied Chemistry (IOSRJAC), ISSN: 2278-5736. Volume 3, Issue 1(Nov. - Dec. 2012), PP 38-45

[13] L.LECREN: Thesis: "Organizations of molecular magnets in inorganic networks and coordination: Synthesis, structure and properties". Doctoral Thesis, University of Bordeaux 1, France, 2006, pp. 76-83.

[14] J. JACOBY: Thesis: "Lipase Mucor miehei immobilized in silicated porous materials: bioreactors for the synthesis of methyl esters from vegetable oils". Doctoral Thesis, Lorraine Doctoral School of Molecular Chemistry and Physics, France, 2013, p 59-89

[15] Engineering Techniques, MeasurementAnalyzes, Analytical Techniques, Thermal Methods of Analysis «article updated from P 1260 published in 2001».

[16] K. S. W. SING : « IUPAC reporting physisorption data for GAS /SOLID systems; with Special Reference to the Determination of Surface Area and Porosity ». Pure \& Appl.Chem.., Vol.54, No.11, pp.22OI 2218, 1982.

[17] X. Wang; K. S. K. Lin; J. C. C. Sheng; "Direct Synthesis and Catalytic Applications of Ordered Large Pore Aminopropyl-Functionalized SBA-15 Mesoporous Materials". J. Phys. Chem. B 2005, 109, 1763-1769.

[18] S.Calmettes: Thesis: "Design of a catalyst by grafting a complex of ruthenium in a confined environment; Application to selective oxidation ". Thesis of Doctorate, University of Lyon, Ecole Normale Supérieure de Lyon, 2008, p. 60-68.

[19] E. ERRAIS: Thesis: "Surface reactivity of natural clays: Study of adsorption of anionic dyes". PhD, University of Strasbourg, France, 2011, p 144. [20] L. MARCOUX: "Study of the physico chemical characteristics of composites Functionalized polymer / mesostructured silica ". Doctoral Thesis, University of LAVAL, Canada, 2013, p 55-71.
[22] A. CHARMOT: Thesis: "New strategies for the insertion of iron oxide particles in organized mesostructures, characterization and analytical activity". Doctoral Thesis, Ecole Supérieure d'Ingénieurs de Poittier, France, 2006, p. 71-74.

[21] N.TOUMI: Thesis: "Synthesis of MicrosMesoporous Composite Materials from ZSM-5 Zeolite". Memoir of Magister, University of ORAN, 2006, 56-59.

[23] S.BADSI and R. ROUIDJA: Thesis: "Synthesis and modification of a mesoporous material type MCM-41 by a series of amines for the adsorption of anionic dyes". Master thesis, University of Science and Technology of Oran Mohamed Boudiaf, 2014, p 31-41.

[24] A. NAIT BAHLOUL and S.SAIDANI: Thesis: "Synthesis and modification of a mesoporous material type MCM-41 by $\mathrm{N}-\mathrm{N}$ dimethyl-n-octylamine: Application to the adsorption of 2,4,6 trichlorophenol". Master thesis, University of Science and Technology of Oran Mohamed Boudiaf, 2013, p 37-45.

[25] Onelia A. Andreo dos Santos, Camila Z. Castellib, Maria Fernanda Oliveira, Ambrósio F. de Almeida Neto and Meuris G. C. da Silva: Thesis: "Adsorption of Synthetic Orange Dye Wastewater in Organoclay". University of MaringáParaná, Brazil VOL. 32, 2013.

[26] S. ACHOUR, N. SEGHAIRI: Thesis: "Possibility of retention of humic substances by adsorption on bentonite". LARHYSS Journal, No. 01, May 2002.

[27] O.Bouras: Thesis: "Adsorption properties of organophilic bridged clays: Synthesis and characterization". Thesis of Doctorate, University of Limoges, 2005.

[28] A.J. Hickey and H.D.C. Smyth, PharmacoComplexity, Outlines in Pharmaceutical Sciences 1, DOI 10.1007 / 978-1-4419-7856-1-2, (C) American Association of Pharmaceutical Scientists 2011.

[29] A.J. Hickey and H.D.C. Smyth, Pharmaco Complexity, Outlines in 5 Pharmaceutical Sciences 1, DOI 10.1007/978-1-4419-7856-1_2, American Association of Pharmaceutical Scientists 2011 [30] Stephen Brunauer, P. H. Emmett, and Edward Teller : « Adsorption of Gases in Multimolecular Layers ». Journal of the American Chemical Society; 1938; 60 ;pp 309 319. 